\title{
Effective complex hydrophobizators for self- cleaning facade materials
}

\author{
Mikhail Bruyako, Natalya Ivashchenko ${ }^{*}$, Kristina Dobrina and Mikhail Moshnikov \\ Moscow State University of Civil Engineering, Yaroslavskoe shosse, 26, Moscow, 129337, Russia
}

\begin{abstract}
In the work presented here, we present the research results of the effect of self-cleaning of surfaces. The methods of its achievement such as photocatalytic and the method of hydrophobization are considered. The known methods for determining the contact angle of wetting are used. An installation for generating a low-temperature nonequilibrium plasma was used to modify a fine aggregate. The contact angles of wetting obtained as a result of the use of various formulations of hydrophobizing suspensions are determined experimentally. A modification of the quartz filling matter was also carried out. Experiments have confirmed the technical effectiveness of hydrophobisers and the enhancement of their properties when dispersed fillers are used. Even higher values of wetting contact angle were achieved using a plasma-modified filling matter.
\end{abstract}

\section{Introduction}

In the design of living, industrial, public buildings and constructions, in addition to the general requirements for space planning decisions, great importance is given to architectural expression. Recently, ventilated facades have been used as an effective solution for outside finish of buildings. The carcass of ventilated facades includes a mount system, a heat-insulating layer and a protective-decorative material. In general, ventilated facades provide a reduction in heat losses and perform protective and decorative functions.

The ecological situation in large cities is declining constantly. This leads not only to the surface fouling and deterioration of the architectural expressions of the facade, but also to the surface deterioration of protective and decorative materials. Controlled at working condition of the facades requires additional economic expenditures connected with planned work related to cleaning the surface of the facades. For this effect, industrial climbers are involved, using in their work a significant amount of detergents based on surface active substances, which after treatment of the surface of buildings fall in the form of aqueous solutions into the sewerage. This leads to additional economic and labor costs.

In this regard, one of the ways to solve the cost problem is to use self-cleaning facades or to give a self-cleaning effect to the surface of traditional materials. This effect is achieved by the following methods:

- photocatalytic method;

- complex hydrophobizators based on finely dispersed inorganic and liquid silicone hydrophobizators.

\footnotetext{
* Corresponding author: ivashenko.nata@mail.ru
} 
The photocatalytic method consists in the imparting hydrophilicity to the surface: the finishing coat attracts water, which when it hits the surface react into a water film. Water in the form of a thin film spreads over the surface and rinses the dirt.

Due to hydrophilic properties, there are no traces of mineral deposits of dried drips after the rain on the surface. Solar energy decomposes the residual dirt.

To enhance the photocatalytic effect, carbon-modified titanium dioxide [1][2].

The principle of self-cleaning with the help of hydrophobizators is to impart superhydrophobicity to the surface of the material. Superhydrophobicity is achieved due to the formation of micro and nano roughness, created by a film based on dispersion or emulsion with the addition of fillers with a size of 0.1 to $200 \mu \mathrm{m}$ [3]. As such dispersed materials, oxides of titanium, aluminum, silicon with a specific surface up to $200 \mathrm{~m} 2 / \mathrm{g}$ can be used [4]. By analogy with the blotchness of the surface of the lotus leaf (Fig. 1), this structure give rise to water droplets on the surface and the removal of mechanical impurities by trapping and removing deposits.

One way to impart hydrophobic properties to surfaces that do not have this property is the treatment with hydrophobisators. Practical interest for the solution of this problem is represented by water-soluble silicon-hydrated hydrophobisators.

It is known that the effect of increasing the hydrophobic properties of surfaces can be realized by means of a complex coating with a hydrophobizator with microdispersed fillers. Or with the help of organo-inorganic complex nanomaterials, which have a superhydrophobic effect, achieved by surfacing titanium or aluminum hydroxide[7].

The microstructure and chemical properties of the solid surface determine the wettability, which is characterized by the wetting angle [8] [9].

From a physical point of view, this is described by the Cassie law, which determines the effective value of the contact angle $\theta \mathrm{c}$ with the fluid in the case of an inhomogeneous surface. This is mathematical expression of the law:

$$
\cos \theta \mathrm{c}=\mathrm{y} 1 \cos \theta 1+\mathrm{y} 2 \cos \theta 2
$$

where $\theta 1$ is the angle of the contact of component 1 present in the composite material, with a fraction of the surface area $y 1$;

$\theta 2$ is the angle of the contact of component 2 present in the composite material, with a fraction of the surface area y2.

The law explains how to increase the value of the contact angle for the surface only by giving it a roughness.

The degree of surface roughness provides a reduction in adhesion between water and the surface of the material and is related to the dispersability of the solids to be removed if they are present in the water, i.e., has a relationship with the size of the particles to be removed. This explains, in particular, the effect desolidization by water, due to the higher adhesion between water and particulate solids than the adhesion between the latter and the surface of the material. The self-cleaning effect is shown in Fig. 1 schematically:

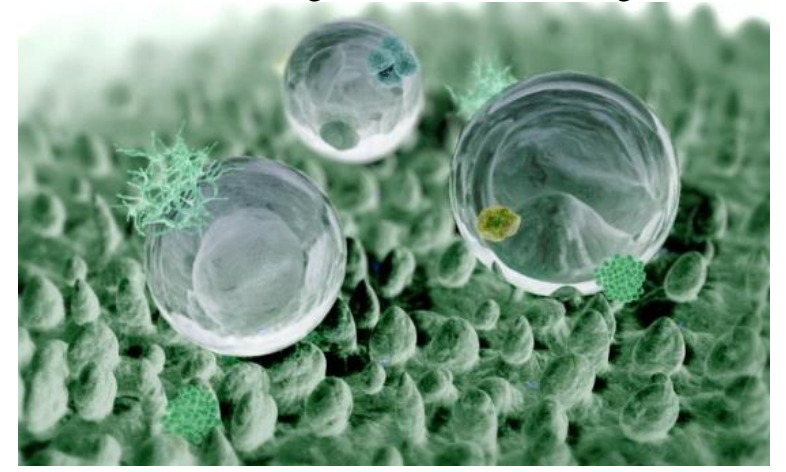

Fig. 1. The effect of self-purification on the example of lotus. 


\section{Methods}

In the work presented here, studies were carried out to determine the effectiveness of hydrophobic suspensions based on liquid hydrophobisators and dispersed fillers. The following domestic brands of organosilicon fluids were chosen as liquid hidrophobistors: Aquasil TU 2229-003-60543126-2014, GKZH 10 and GKZh 11 TU 6-02-696-76; disperse micro fillers $\mathrm{TiO} 2$ and $\mathrm{Al} 2 \mathrm{O} 3$.

Suspensions were prepared in keeping with the formulations given in Table 1, on a high-speed mixer with a shaft rotation speed of $1000 \mathrm{rpm}$. After that, the surpensions were applied by roller to the surface of the samples.

For the preparation of samples based on a cement-sand mortar, a white Portland cement of CEM I $52.5 \mathrm{~N}$ (Cimsa, Turkey). The superplasticizer C-3 is used as the plasticizing agent. It is inexpensive and versatile. The work used sand from the quarry "Putilovo -2". The work used main water.

Table 1. Composition of coatings applied.

\begin{tabular}{|c|c|c|c|c|c|c|}
\hline $\begin{array}{l}\text { Mar } \\
\text { king }\end{array}$ & $\begin{array}{c}\text { KE } \\
\text { 30-04, } \\
\text { weight } \\
\text { part }\end{array}$ & $\begin{array}{l}\text { GKZH 10, } \\
\text { weight part }\end{array}$ & $\begin{array}{l}\text { GKZH 11, } \\
\text { weight part }\end{array}$ & $\begin{array}{c}\text { Aquasil, } \\
\text { weight part }\end{array}$ & $\begin{array}{c}\text { Filler Al2O3, } \\
\text { weight part }\end{array}$ & $\begin{array}{l}\text { Filler TiO2, } \\
\text { weight part }\end{array}$ \\
\hline 1 & 100 & & & & 0 & \\
\hline 2 & 100 & & & & 1 & \\
\hline 3 & 100 & & & & 2 & \\
\hline 4 & 100 & & & & 4 & \\
\hline 5 & 100 & & & & & 1 \\
\hline 6 & 100 & & & & & 2 \\
\hline 7 & 100 & & & & & 4 \\
\hline 8 & & 100 & & & 0 & \\
\hline 9 & & 100 & & & 1 & \\
\hline 10 & & 100 & & & 2 & \\
\hline 11 & & 100 & & & 4 & \\
\hline 12 & & 100 & & & & 1 \\
\hline 13 & & 100 & & & & 2 \\
\hline 14 & & 100 & & & & 4 \\
\hline 15 & & & 100 & & 0 & \\
\hline 16 & & & 100 & & 1 & \\
\hline 17 & & & 100 & & 2 & \\
\hline 18 & & & 100 & & 4 & \\
\hline 19 & & & 100 & & & 1 \\
\hline 20 & & & 100 & & & 2 \\
\hline 21 & & & 100 & & & 4 \\
\hline 22 & & & & 100 & 0 & \\
\hline 23 & & & & 100 & 1 & \\
\hline 24 & & & & 100 & 2 & \\
\hline 25 & & & & 100 & 4 & \\
\hline 26 & & & & 100 & & 1 \\
\hline 27 & & & & 100 & & 2 \\
\hline 28 & & & & 100 & & 4 \\
\hline
\end{tabular}

After a day, the contact angle was determined. There are several techniques for its calculation [11] [12]. There is technique, described in the literature in this case. 


\section{Results}

The value of the contact angle of wetting of surfaces depending on the quantitative and qualitative composition of hydrophobic suspensions is given in Table 2.

Table 2. The value of the contact angle of wetting for various hydrophobic surfaces.

\begin{tabular}{|c|c|c|c|}
\hline Marking & Contact angle, ${ }^{\circ}$ & $\begin{array}{l}\text { Visual effect self- } \\
\text { cleaning }\end{array}$ & $\begin{array}{c}\text { Weatherstain of the } \\
\text { material }\end{array}$ \\
\hline Control & 53 & no visual effect & - \\
\hline 1 & 65 & thin & - \\
\hline 2 & 70 & thin & thin \\
\hline 3 & 90 & thin & have \\
\hline 4 & 95 & thin & have \\
\hline 5 & 75 & thin & thin \\
\hline 6 & 98 & thin & thin \\
\hline 7 & 102 & thin & have \\
\hline 8 & 80 & thin & - \\
\hline 9 & 85 & thin & - \\
\hline 10 & 104 & medium & thin \\
\hline 11 & 126 & visible self-cleaning & have \\
\hline 12 & 89 & thin & thin \\
\hline 13 & 110 & medium & thin \\
\hline 14 & 130 & have & have \\
\hline 15 & 66 & thin & - \\
\hline 16 & 68 & thin & thin \\
\hline 17 & 95 & thin & thin \\
\hline 18 & 99 & medium & have \\
\hline 19 & 72 & thin & thin \\
\hline 20 & 102 & medium & thin \\
\hline 21 & 116 & medium & have \\
\hline 22 & 75 & medium & - \\
\hline 23 & 87 & medium & - \\
\hline 24 & 124 & visible self-cleaning & - \\
\hline 25 & 134 & visible self-cleaning & thin \\
\hline 26 & 94 & severe & - \\
\hline 27 & 118 & visible self-cleaning & - \\
\hline 28 & 148 & visible self-cleaning & - \\
\hline
\end{tabular}

To increase the self-cleaning effect, when materials are produced in the factory, a material based on plasma-modified fine filling matters should be used.

Processing of quartz sand in a low-temperature nonequilibrium plasma leads to a modification of the characteristics of its surface and near-surface layers. So, the use of plasma for surface modification is known, which allows making local changes in the properties of the surface being treated [12].

In this connection, studies have also been carried out to determine the effect of complex superhydrophobic coatings on samples with a previously modified fine quartz aggregate.

Modification of mineral fillers ( $\mathrm{SiO} 2)$ was carried out in a facility for generating a lowtemperature nonequilibrium plasma. $\mathrm{SiO} 2$ (sand) was treated with a low-temperature nonequilibrium plasma in a barrier discharge, after which the chemical composition of the treated and untreated in sand was analyzed on a Senterra Raman spectrometer. 
As a result of the action of low-temperature plasma, amorphization and structural changes in the surface occur (Table 3).

Table 3. Change in the roughness of quartz sand.

\begin{tabular}{|c|c|c|c|c|c|c|}
\hline \multirow{2}{*}{ 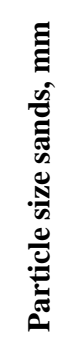 } & \multirow{2}{*}{ 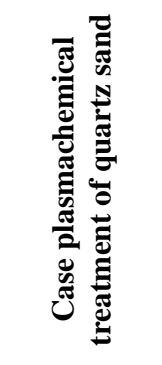 } & \multirow{2}{*}{ 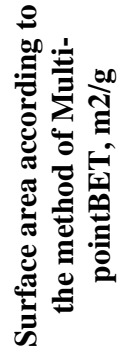 } & \multirow{2}{*}{$\begin{array}{l}\text { Correlation } \\
\text { factor } R\end{array}$} & \multicolumn{3}{|c|}{$\begin{array}{l}\text { Pore distribution quartz sand } \\
\text { to the method of BJH }\end{array}$} \\
\hline & & & & 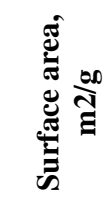 & 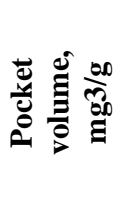 & 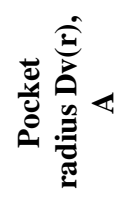 \\
\hline 0.15 & - & 2.098 & 0.995 & 0.785 & 0.15 & - \\
\hline 0.15 & $\begin{array}{l}\text { To barrier } \\
\text { dischare }\end{array}$ & 2.235 & 0.997 & 0.858 & 0.15 & $\begin{array}{c}\text { To } \\
\text { barrier } \\
\text { dischare }\end{array}$ \\
\hline
\end{tabular}

It can be seen from Fig. 2 that when processing quartz sand in a low-temperature nonequilibrium plasma, a peak of $465.07 \mathrm{~cm}-1$ disappears, indicating the presence of $\mathrm{SiO} 2$. This is apparently due to the change in the crystal lattice of the substance and the transition of the sand surface to the amorphyrin state.

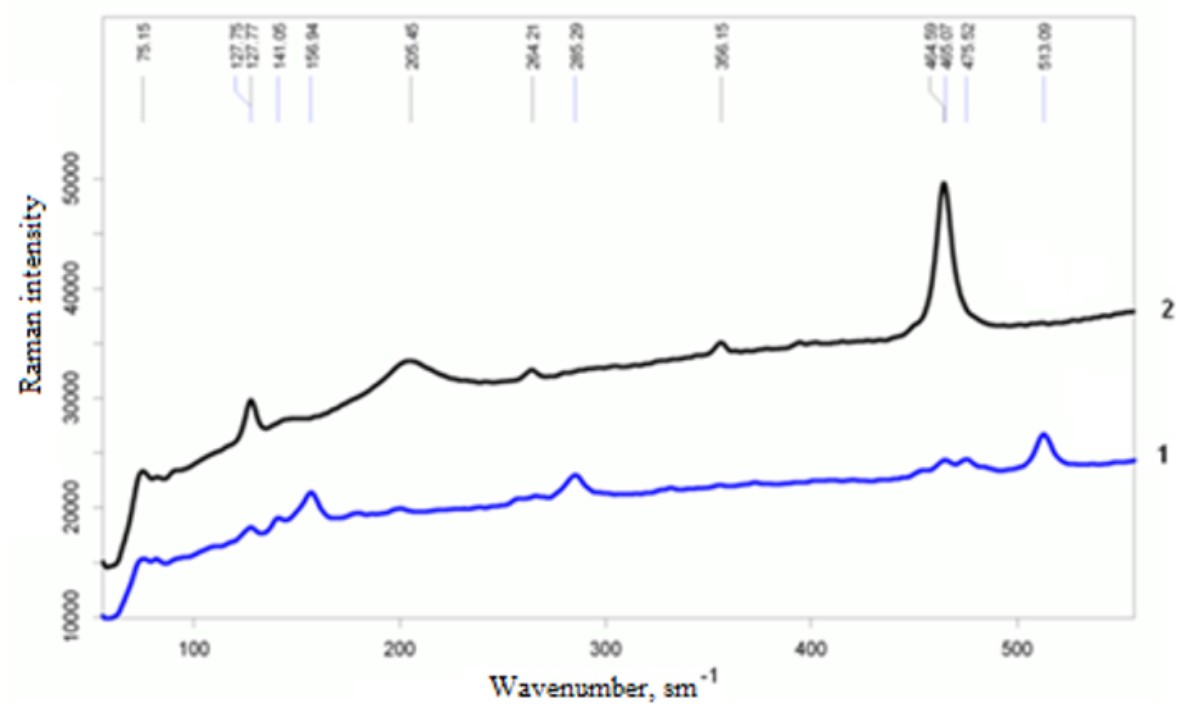

Fig. 2. Raman scattergram of light.

\section{Discussion}

The most effective organosilicon hydrophobisers are Aquasil and GKZh10.

The increase in the wetting contact angle depends both on the quantitative composition of the microparticles and on the chemical nature of the hydrophobisers. The effectiveness 
of the coating depends on this, too. The greatest effect of imparting hydrophobicity to materials was shown by suspensions based on the silicone hydrophobizer Aquasil, and the efficiency of dispersed fillers $\mathrm{TiO} 2$. The greatest value of contact angle of wetting was achieved on suspensions based on Aquasil and GKZh10 in combination with titanium dioxide. At the same time, the maximum value of contact angle of wetting was achieved on a surface treated with a suspension based on 100 parts by weight of Aquasil and 4 parts by weight of $\mathrm{TiO} 2$ (see Table 1, 2).

Aquasil and $\mathrm{TiO} 2$ do not affect the appearance of the treated material. The use of suspensions based on Aquasil and titanium dioxide to give superhydrophobicity to the surfaces being treated is most appropriate.

Use of dispersed micronutrients to impart surface hydrophobicity to liquid kreeneorganic hydrophobisers allows to increase the effectiveness of protective building materials. For example, the introduction of titanium oxide in the hydrophobizer brand Aquasil allows you to increase the contact angle of wetting.

\section{Conclusions}

The results of the study make it possible to judge the possibility of achieving a self-cleaning effect in materials with a superhydrophobic surface. The wetting angle of which reaches a value of 165 degrees. An increase in the wetting contact angle from 10 to 13 percent was found with the surface of samples containing a modified quartz aggregate.

For the treatment of existing facades, it is effective to use complex water repellents. The greatest effect was shown by coatings based on suspensions containing more fillers (4 parts by weight). To obtain self-cleaning materials in the factory, use a plasma-modified fine aggregate or a combination of the two methods to obtain maximum results.

This research was supported by The Head Regional Shared Research Facilities of the Moscow State University of Civil Engineering.

\section{References}

1. Patent RU $2380318 \mathrm{C} 2$

2. Patent RU $2377267 \mathrm{C} 2$

3. Patent RU $2246514 \mathrm{C} 2$

4. Patent RU $2318781 \mathrm{C} 2$

5. Patent RU $2331532 \mathrm{C} 2$

6. Vinogradov A.V. Sol-gel synthesis of titanium dioxide based films possessing highly ordered channel structure. / Vinogradov A.V., Agafonov A.V. // Abstract. Intern. Conf. «Sol-Gel 2009». - Recife, Brazil. -23-27 august. 2009.

7. Zhang CY, Cai HG, Chen B et al (2008) Chin J Catal 29:1

8. Chang KC, Chen YK, Chen H (2008) Surf Coat Technol 202:3822

9. Kiselev MG, Savich VV, Pavch TP. Messenger BNTU No.1, 2006 c.38-41

10. United States patent 5268733, G 01 B 11/26; G 01 N 13/02, 1993

11. United States patent 4688938, G 01 B 11/26

12. A.M. Efremov, V.I. Svettsov, V.V. Rybkin. Vacuum-plasma technologies, Ivanovo, 2006, C.8. 